\title{
Face Injury
}

National Cancer Institute

\section{Source}

National Cancer Institute. Face Injury. NCI Thesaurus. Code C34603.

Trauma to the face. 